\title{
LIMIT SETS IN PRODUCT OF SEMI-DYNAMICAL SYSTEMS
}

\section{RAMJEE PRASAD BHAGAT}

(Received 12 November 1996 and in revised form 9 March 1998)

\begin{abstract}
Continuing the study of the properties of Poisson stability and distality [4], we mention the conditions under which $\Omega_{x}(x)=\Pi \Omega_{\alpha}\left(x_{\alpha}\right), \alpha \in I$ and thus, the product of Poisson stable motions remains Poisson stable in the product system.
\end{abstract}

Keywords and phrases. Semi-dynamical system, Lagrange stability, distality, limit sets.

1991 Mathematics Subject Classification. 47H10, 54H25.

1. Introduction. We deal mainly with the product of $w$-limit sets in the product space of semi-dynamical systems (s.d.s.). In [1], Prem Bajaj has shown that the product of semi-dynamical systems is a semi-dynamical system. He has also shown that $\Pi \Omega_{\alpha}\left(x_{\alpha}\right), \alpha \in I$ contains the $w$-limit set $\Omega_{x}(x)$ of $x$ in the product system. In general, equality does not hold in the above. Indeed $\Omega_{x}(x)$ may be empty. He has given two theorems: one in which $\Omega_{x}(x)$ is nonempty and the other indicating a case of equality viz. Theorems 2.3 and 2.4 .

In this paper, continuing the study of the properties of Poisson stability and distality [4], we mention the conditions under which $\Omega_{x}(x)=\Pi \Omega_{\alpha}\left(x_{\alpha}\right), \alpha \in I, x=\left\{x_{\alpha}\right\}$ and therefore, the product of Poisson stable motions, under these conditions, is Poisson stable.

\section{Definitions and notations}

DEFINITION 2.1. A continuous mapping $\pi: X \times \mathbb{R}^{+} \rightarrow X$ on a topological space $X$ is said to define a semi-dynamical system $(X, \pi)$ if $\pi(x, 0)=x$ and $\pi(\pi(x, t), s)=$ $\pi(x, t+s)$ for every $x \in X$ and $t, s \in \mathbb{R}^{+}$. ( $\mathbb{R}^{+}$denotes the set of nonnegative reals.)

DEFINITION 2.2. Let $\left(X_{\alpha}, \pi_{\alpha}\right), \alpha \in I$ be a family of dynamical systems. Let $X=$ $\Pi X_{\alpha}$ be the product space. Let $x \in X$ and $x=\left\{x_{\alpha}\right\}$. Define a map $\pi$ from $X \times \mathbb{R}$ into $X$ by $\pi\left(x_{\alpha} t\right)=\left(x_{\alpha} t\right), \alpha \in I$, then $(X, \pi)$ is a dynamical system. The dynamical system $(X, \pi)$, obtained above, is called the direct product or the product of the family $\left(X_{\alpha}, \pi_{\alpha}\right), \alpha \in I$.

We take the usual definitions of positive limit set $\Omega_{x}$, positive distal, positive Poisson stable, and positive Lagrange stable motions. As usual, we drop the word positive and we use the notations of $[1,4]$.

\section{Main results}

Proposition 3.1. Let $\left(X_{\alpha}, \pi_{\alpha}\right), \alpha \in I$, be a family of $\{$ Lagrange stable $\}\{$ distal $\}$ s.d.s. 
and $(X, \pi)$ the product s.d.s. Let $x \in X$ and $x=\left\{x_{\alpha}\right\}$, then $(X, \pi)$ is $\{$ Lagrange stable $\}$ $\{$ distal $\}$.

Proposition 3.2. If a Lagrange stable motion is Poisson stable and distal, then $\operatorname{ClY}(x)=\Upsilon(x)=\Omega_{x}$.

Proof. The proof follows from [4, Thm. 2.1].

THEOREM 3.3. Let $\left(X_{\alpha}, \pi_{\alpha}\right), \alpha \in I$, be a family of dynamical systems and $(X, \pi)$ the product of the dynamical systems. Let $x \in X$ and $x=\left\{x_{\alpha}\right\}$. Then $\Omega_{x}(x) \subseteq \Pi \Omega_{\alpha}\left(x_{\alpha}\right)$, where $\Omega_{\alpha}\left(x_{\alpha}\right)$ is the positive limit set of $x_{\alpha}$ in the dynamical systems $\left(X_{\alpha}, \pi_{\alpha}\right)$. (The two $\pi$ 's have distinct meanings according to the context.)

Since, in general, the equality does not hold and $\Omega_{x}$ may be empty, the Poisson stability in the constituent dynamical system may be lost from the product of the dynamical systems. Here, we find the conditions under which $\Omega_{x}(x)=\Pi \Omega_{\alpha}\left(x_{\alpha}\right), \alpha \in I$ and thus, the product of Poisson stable motions remains Poisson stable in the product system.

THEOREM 3.4. If a compact motion is Poisson stable and distal, then it is a compact recurrent motion.

Proof. Let the motion $\pi(x, t)$ be Poisson stable and distal, then its trajectory $\Upsilon(x)$ is closed. Therefore,

$$
\Upsilon(x)=\operatorname{ClY}(x)=\Omega_{x} .
$$

As the motion is compact, each of the above sets is compact and minimal and thus, by Birkhoff recurrence theorem, $\pi(x, t)$ is compact and recurrent.

THEOREM 3.5. Let $(X, \pi)$ be a semi-dynamical system. Let $\pi$ be a Lagrange stable, then $\pi$ is distal if and only if, for every net $t_{i}$ in $\mathbb{R}^{+}$, the phase space

$$
X=\left\{z \in X: x t_{j} \longrightarrow z \text { for some } x \in X \text { and some subnet } t_{j} \text { of } t_{i}\right\}
$$

[2, Thm. 2.6].

THEOREM 3.6. Let $(X, \pi)$ be Lagrange stable and distal s.d.s. then every net in the trajectory $Y(x)$ of the Poisson stable motion $\pi(x, t)$ is a Cauchy net.

Proof. Let $Y(x)$ be the trajectory of the Poisson stable motion $\pi(x, t)$ in s.d.s. $(X, \pi)$ which is Lagrange stable and distal. Let $x t_{n}$ be a net in $Y(x)$ which is compact (Proposition 3.2). Therefore, $x t_{n}$ has a subnet, say $x t_{m}$ with $x t_{m} \rightarrow z$, i.e., $z$ is a cluster point of $x t_{n}$. Hence, $x t_{n}$ is a Cauchy net.

THEOREM 3.7. Let $\left(x_{\alpha}, \pi_{\alpha}\right), \alpha \in I$, be a family of Lagrange stable and distal s.d.s. and $(X, \pi)$ be the product s.d.s. Let $x \in X$ and $x=\left\{x_{\alpha}\right\}$. A motion $\pi(x, t)$ is Poisson stable in $(X, \pi)$ if and only if $\pi_{\alpha}\left(x_{\alpha}, t\right)$ is Poisson stable in $\left(X_{\alpha}, \pi_{\alpha}\right)$ for each $\alpha \in I$.

Proof. Let $\left(x_{\alpha}, \pi_{\alpha}\right), \alpha \in I$, be a Lagrange stable and distal s.d.s. Let $\pi\left(x_{\alpha}, t\right)=x_{\alpha} t$ be a Poisson stable motion in $\left(X_{\alpha}, \pi_{\alpha}\right), \alpha \in I$, then its trajectory $\Upsilon_{\alpha}\left(x_{\alpha}\right)$ is compact and the net $x_{\alpha} t_{n}, \alpha \in I$, is a Cauchy net in $\Upsilon_{\alpha}\left(x_{\alpha}\right)$ (Theorem 3.6). Now, the Cauchy 
nets $x_{\alpha} t_{n}, \alpha \in I$ yield the Cauchy net $x t_{n}$ in $Y(x)$ in $(X, \pi)$ [3, p. 194]. As the product of compact sets is a compact set, $Y(x)$ is compact and $x t_{n}$ is a net in compact $Y(x)$. Thus, it has a subnet $x t_{m} \rightarrow z$, i.e., $z$ is a cluster point of $x t_{n}$. Hence, $x t_{n}$ is frequently in every neighborhood $U$ of $z$. Given a neighborhood $U$ of $z$ for every $i \in A$, there is a $j \in A, i \geq J$ such that $x t_{i} \in U$ however $t_{i} \rightarrow+\infty$. Hence, $\pi(x, t)$ is Poisson stable. The converse follows from [3, Thm. 25, p. 194] which states that a net in the product is a Cauchy net if and only if its projection into each coordinate space is a Cauchy net.

THEOREM 3.8. Let $\left(X_{\alpha}, \pi_{\alpha}\right), \alpha \in I$, be a family of Lagrange stable distal s.d.s. Let $x \in X, x=\left\{x_{\alpha}\right\}$, and $(X, \pi)$ the product s.d.s. Let $\Upsilon_{\alpha}\left(x_{\alpha}\right), \alpha \in I$, be the product of trajectries. Then $\Pi \Upsilon_{\alpha}\left(x_{\alpha}\right)=\Upsilon(x)$. Moreover,

$$
\Pi \Omega_{\alpha}\left(x_{\alpha}\right)=\Omega_{x}(x)
$$

Proof. Since each $\Upsilon_{\alpha}\left(x_{\alpha}\right), \alpha \in I$, is closed and compact,

$$
\begin{aligned}
\operatorname{Cl\Pi \Upsilon }_{\alpha}\left(x_{\alpha}\right) & =\Pi \operatorname{ClY}_{\alpha}\left(x_{\alpha}\right)=\operatorname{ClY}(x), \\
\Pi \Upsilon_{\alpha}\left(x_{\alpha}\right) & =\Upsilon(x) .
\end{aligned}
$$

Moreover,

$$
\Pi \Omega_{\alpha}\left(x_{\alpha}\right)=\Omega_{x}(x)
$$

\section{REFERENCES}

[1] P. N. Bajaj, Products of semi-dynamical systems, Recent advances in topological dynamics (Proc. Conf., Yale Univ., New Haven, Conn., 1972; in honor of Gustav Arnold Hedlund) (Berlin), Lecture Notes in Math, vol. 318, Springer, Verlag, 1973, pp. 23-29. MR 53 1549. Zbl 258.54042.

[2] N. P. Bhatia and M. Nishihama, Distal semidynamical systems, Dynamical systems (Proc. Internat. Sympos., Brown Univ., Providence, R.I., 1974) (New York), vol. II, Academic Press, 1976, pp. 187-190. MR 58 31006. Zbl 359.54033.

[3] J. L. Kelley, General topology, Springer-Verlag, New York, Berlin, 1975. MR 516681. Zbl 518.54001.

[4] S. S. Prasad and A. Kumar, Stable P and distal dynamical systems, Internat. J. Math. Math. Sci. 7 (1984), no. 1, 181-185. MR 85e:34045. Zbl 562.54061.

Bhagat: Department of Mathematics, A. S. College Bikramganj, Pin 802212, Rohtas, BIHAR, INDIA 


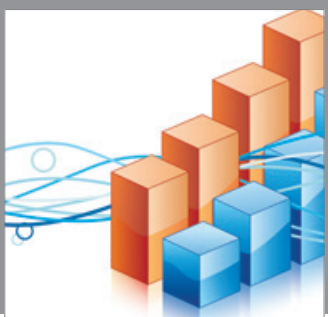

Advances in

Operations Research

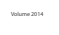

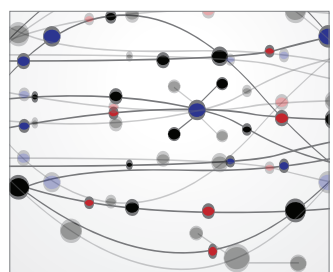

\section{The Scientific} World Journal
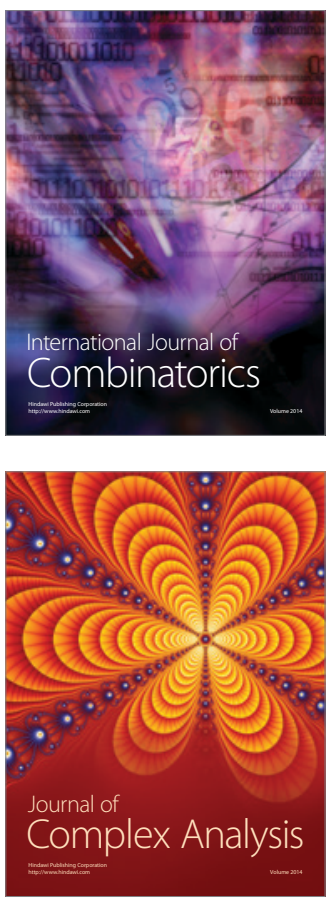

International Journal of

Mathematics and

Mathematical

Sciences
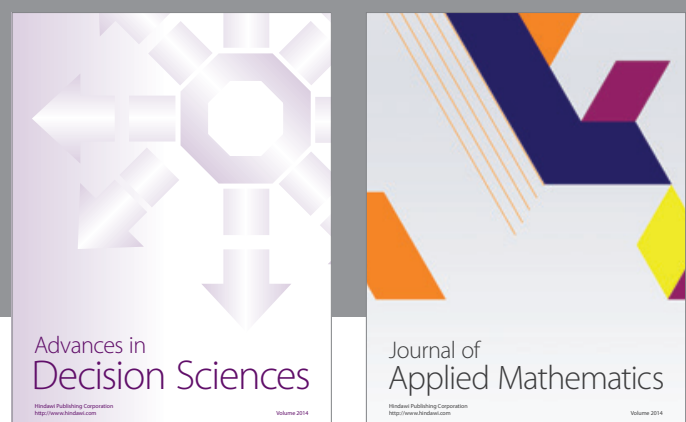

Journal of

Applied Mathematics
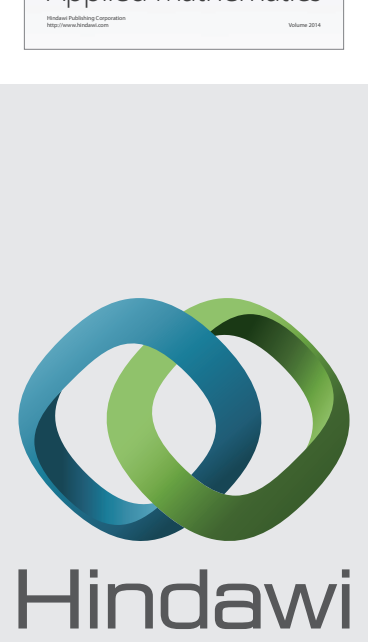

Submit your manuscripts at http://www.hindawi.com
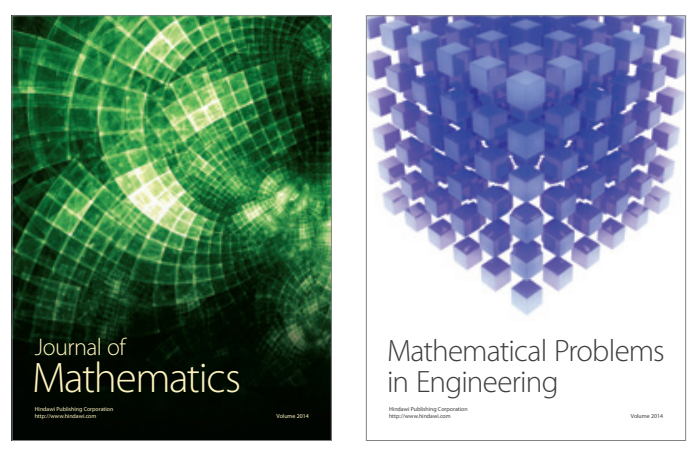

Mathematical Problems in Engineering
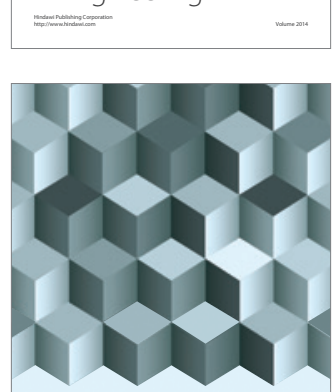

Journal of

Function Spaces
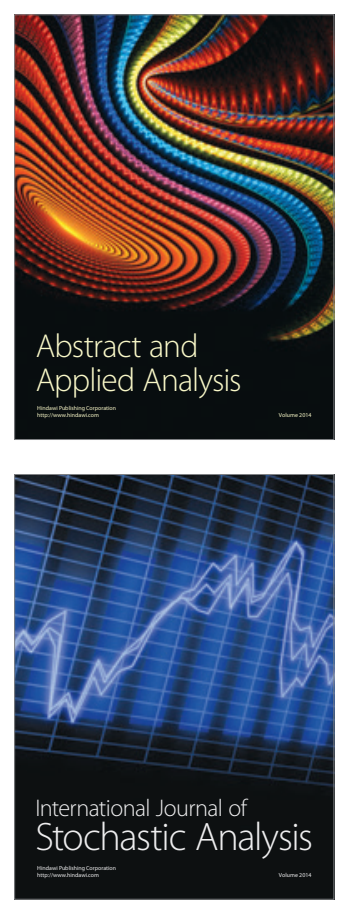

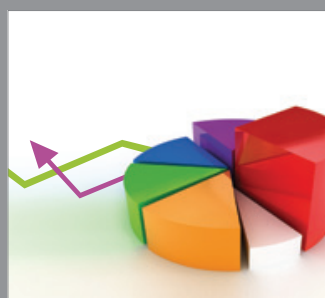

ournal of

Probability and Statistics

Promensencen
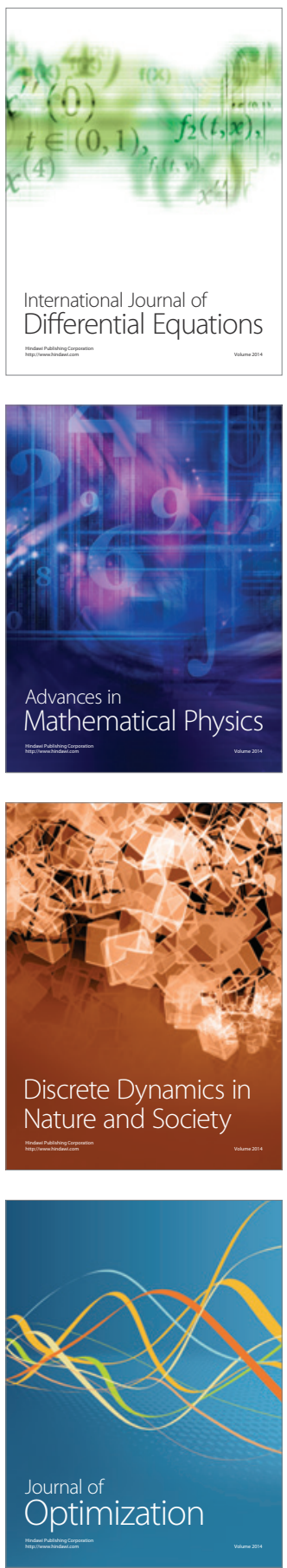